\title{
A Systematic Review of Clinical Studies Using VEGF Inhibitors in the Treatment of Macular Edema from Diabetic Retinopathy
}

\author{
Karim Diab ${ }^{1}$, Swati Chavda ${ }^{1}$, Nathan Gorfinkel' ${ }^{1}$, Francie Si², Brad Dishan ${ }^{3}$, William Hodge ${ }^{2 *}$ \\ ${ }^{1}$ Western University, London, Canada \\ ${ }^{2}$ Ivey Eye Institute, Western University, London, Canada \\ ${ }^{3}$ Library Service, St. Joseph's Hospital Health Care, London, Canada \\ Email: *William.Hodge@sjhc.Iondon.on.ca
}

Received 24 November 2015; accepted 1 February 2016; published 4 February 2016

Copyright (C) 2016 by authors and Scientific Research Publishing Inc.

This work is licensed under the Creative Commons Attribution International License (CC BY). http://creativecommons.org/licenses/by/4.0/

(c) (i) Open Access

\begin{abstract}
Purpose: To synthesize the present clinical evidence of efficacy and adverse events of commonly used anti-VEGF drugs for Diabetic Macular Edema. Methods: A systematic review was undertaken from the Medline, Biosis, CINAHL, Cochrane and Web of Science databases. Grey literature that consisted of lectures, seminars and conferences was also retrieved. The cut-off date was January 1 2014. A two-stage screening process was undertaken followed by a data extraction stage using the systematic review software EPPI. These were done by two reviewers. Heterogeneous meta-analysis was performed on the primary outcome which was change in macular thickness from baseline after injection. Side effects were tabulated. Results: From 846 articles that were initially screened, 18 papers were included in the data extraction stage. For all anti-VEGF treatments, the average decrease in macular thickness was 114.4 microns (95\% CI: 66.8 - $162 \mu \mathrm{M}$ ). The average decrease in thickness from Lucentis $(161.9 \mu \mathrm{M})$ was larger than that for Avastin $(96.5 \mu \mathrm{M})$ but this was not statistically significant $(p=0.23)$. The most common complications were vitreous hemorrhage, endophthalmitis and retinal detachment. Vision threatening complications were rare but were reported regularly. Conclusions: The synthesized clinical evidence to date supports both of these treatments as efficacious and safe for diabetic macular edema (DME). There is a trend toward greater efficacy for Lucentis over Avastin but this is not statistically significant and will need a head-to-head RCT to assess accurately.
\end{abstract}

\section{Keywords}

Diabetic Retinopathy, VEGF Inhibitors, Macular Edema, Intraocular Injections, Systematic Review

\footnotetext{
${ }^{*}$ Corresponding author.
}

How to cite this paper: Diab, K., Chavda, S., Gorfinkel, N., Si, F., Dishan, B. and Hodge, W. (2016) A Systematic Review of Clinical Studies Using VEGF Inhibitors in the Treatment of Macular Edema from Diabetic Retinopathy. Open Journal of Ophthalmology, 6, 12-19. http://dx.doi.org/10.4236/ojoph.2016.61003 


\section{Introduction}

There is a major rise in the percentage of individuals with diabetes in the global population. This growing number is expected to reach 366 million individuals by 2030 compared to 171 million individuals with the condition in the year 2000 [1]. Most of these cases in the western world are attributed to a change in lifestyle and an increase in obesity and longevity [1]. Patients with diabetes have a diverse set of complications that range from microvascular to macrovascular complications. Microvascular complications are retinopathies, nephropathies and neuropathies while macrovascular complications include myocardial infarctions, hypertension and peripheral arterial diseases [2]. In this paper, we focus on damages caused to the retina by the complications associated with diabetes mellitus; the leading cause of vision loss in working-age individuals worldwide [3].

Vascular Endothelial Growth Factor (VEGF) is a hormone normally liberated by cells in the body in case of a wound or an infection in order to form new blood vessels and restore oxygen levels in the damaged tissue. Symptoms caused by diabetes such as hyperglycemia, oxidative stress, hypoxia and inflammatory reactions increase the expression of VEGF [4]. Also, retinal endothelial cells have a high number of VEGF receptors that increase in patients with diabetes [4]. VEGF contribute significantly to the development of Diabetic macular edema (DME) by affecting the tight junctions of endothelial cells in the retina by increasing leukocyte count and these leukocytes are known to cause leakage [3]. This causes the breakdown of the blood-retinal barrier and the accumulation of fluid in the macula [5] which in turn will result in reduced or distorted vision. In addition, VEGF is at least partly responsible for the formation of new blood vessels in the retina (proliferative diabetic retinopathy).

One of the treatments of DME is the injection of antibodies that specifically target VEGF in patients suffering from DME. Three anti-VEGF agents are currently commercially available: Pegaptanib (Macugen, OSI/Eyetech, Melville, NY, USA), Ranibizumab (Lucentis, Genentech, Inc., South San Francisco, CA, USA) and Bevacizumab (Avastin, Genentech, Inc.)

Lucentis and Avastin are humanized antibodies specific to all isoforms of VEGF that are used commonly clinically for intraocular vascular disorders. Although Lucentis is approved by the FDA, Avastin is still undergoing clinical trials to determine whether it is safe and effective for the treatment of DME and other pathologies of the retina. Avastin is primarily an anti-cancer drug but it is used off-label by physicians to treat DME because of its low cost and availability [3]. One of the reasons that Avastin's safety as a treatment for ophthalmic conditions is still being considered is the drug's side effects when used as an anti-cancer drug. Trials of Avastin for Age-related Macular Degeneration (AMD) patients rather than cancer patient presented hypertension as a side effect though occlusive stroke was also seen [6]. However these trials were made on AMD patients who tend to be of an older age group than patients of DME.

Side effects of these anti-VEGF drugs for intraocular conditions are usually rare and procedure related rather than reactions to the medication [3]. Some procedure related outcomes consist of endophthalmitis, retinal detachment and lens injury. DME patients treated with both Avastin and Lucentis have shown significant improvements in vision [3]. A comparative study of Avastin against Lucentis needs to be done in order to evaluate the best candidate for DME treatment. At that point, we will be able to evaluate all the possible outcomes and side effects of each drug along with their costs.

In this study, we are synthesizing all of clinical research results to date of anti-VEGF drugs for DME via a systematic review and meta-analysis.

\section{Methods}

The search for relevant literature was conducted based on the research question "How do Avastin and Lucentis compare to each in terms of outcomes and complications for the treatment of diabetic macular edema"? This research question along with the subsequent keywords used in the different databases was defined with the help of clinical content experts and an information specialist. Multiple synonyms were created for VEGF inhibitors and macular edema treatment.

Relevant literature published in English was obtained using an exhaustive search from the Medline, Biosis, CINAHL, Cochrane and Web of Science databases. Grey literature that consisted of lectures, seminars and conferences was also retrieved. These included abstracts from the American Academy of Ophthalmology, Association for Research in Vision and Ophthalmology, Canadian Ophthalmology Society and European Association of Ophthalmology. The cut-off date was January 1, 2014. No beginning date was specified. The monograph results 
were then inserted into EPPI, a systematic review software program. Duplicates were then removed using EPPI after a bibliographic record was made. Literature was then passed through two levels of screening followed by a data extraction level. Papers had to be included after each level of screening in order for data to be extracted from them. EPPI records whether a publication is included or excluded after each level of screening. The software also records the reason why the paper was excluded.

The first level of screening determined the eligibility of the paper based on the title and the abstract. This level excluded papers that did not focus on patients with diabetic retinopathy. Experimentations on animals were excluded. In addition, research papers that conducted the study on patients under the age of 18 were excluded. Papers that did not examine the effects of Avastin or Lucentis as a treatment were also excluded. Finally, the study had to be conducted in an industrialized country for reasons of generalizability.

The second level of screening involved reviewing the full paper in EPPI in order to determine its eligibility. In this level, papers that were not primary research studies were excluded. Hence, surveys, editorial comments, small case studies, conferences, meetings and systematic reviews were excluded. A sample size of 20 patients or more was necessary in order for the paper to be included. Also, the paper needed to examine the effect of one of the two anti-VEGF drugs on patients with diabetic retinopathy to be included. Both the first and the second level of screening were conducted by multiple reviewers but at least two had to review each paper. Disagreements in the decision to include or exclude a paper were noted and a conclusion was reached by consensus if possible and if not by a third party adjudicator (WGH).

During data extraction, the quality of included articles and risk of bias was assessed by multiple reviewers using the Downs and Black instrument. Both randomized controlled trials and observational studies were assessed using this instrument.

Data was extracted from the included articles and organized using the EPPI software. This data included demographic data, treatment data, outcome data and side effect data. All data was collected on a premade data collection sheet in EPPI. For statistical analysis, the final data was imported into the statistical software STATA (v 14 College Station Texas) for analysis. For outcomes where the data was sufficiently homogenous, a meta-analysis was performed for the defined outcomes. A pooled weighted mean difference was used as the primary outcome. Due to study heterogeneity, the pooled effects were assumed to follow a random effects model (DerSimonian-Laird). Formal meta-analysis was performed for the outcome of macular thickness change from baseline after anti-VEGF injection but was not possible for adverse events.

The Begg test was used to check for publication bias after graphing the potential bias via Funnel plots. Meta-regressions were performed for study level and clinical level variables to see if confounders had influenced the outcomes studied. Multiple comparisons in the meta-regression were adjusted by using a Monte Carlo simulation of 20,000 trials.

\section{Results}

The PRISMA diagram for this study is shown in Figure 1. 846 articles were sent through level I abstract screening followed by 402 that went through full text level II screening. Data extraction occurred on a final sample of 18 articles [7]-[24] that met all inclusion criteria. All of these 18 articles reported on either macular thickness change after injection, adverse events or both. Of the 18 articles included, 11 were randomized clinical trials, 1 was an observational study and 6 were case series. The Downs and Black checklist is a 27 point checklist that assesses study quality. The average score in studies used for this review was 20.6. The range was 13 to 27.

Table 1 provides the baseline data from the studies. The average sample size from the studies was 188 with a little under $50 \%$ being female. Table 2 reveals the treatment characteristics. There were on average 2.66 anti VEGF injections per treatment over an average span of 86 days and patients were followed an average of 317 days from onset of macular edema.

Figure 2 and Figure 3 provide the weighted mean differences Forest Plots from all studies. Figure 2 is the Forest plot of all anti-VEGF drug treatment on macular thickness. As can be seen all but one study demonstrated a significant decrease in macular thickness after treatment compared to before treatment. The average decrease in macular thickness was 114.4 microns (95\% CI: 66.8 - $162 \mu \mathrm{M}$ ). Figure 3 is the Forest plot of anti VEGF treatment stratified by type of VEGF inhibitor. As can be seen from the Forest plot, the average decrease in thickness from Lucentis $(161.9 \mu \mathrm{M})$ was larger than that for Avastin $(96.5 \mu \mathrm{M})$ but this was not statistically 


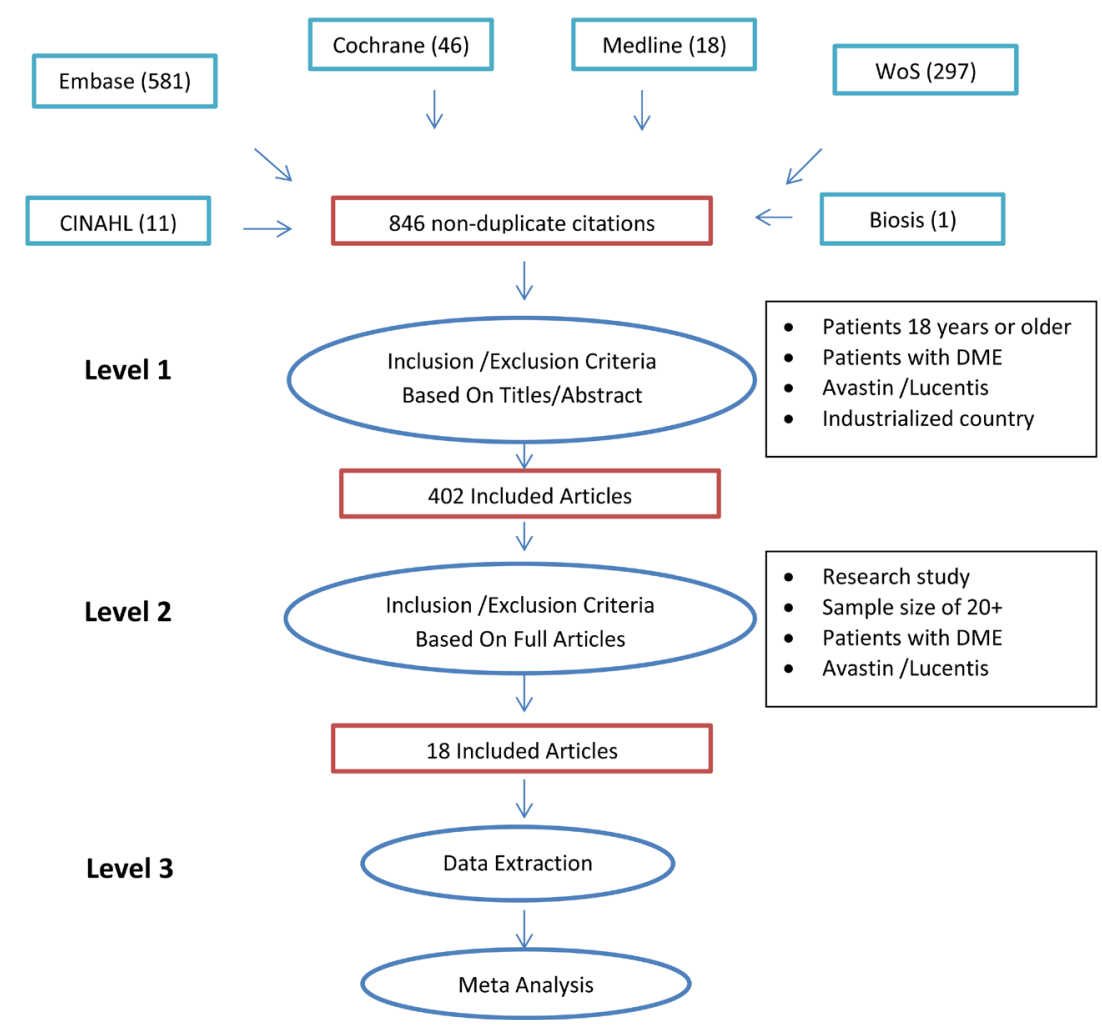

Figure 1. The PRISMA diagram.

Study

ID

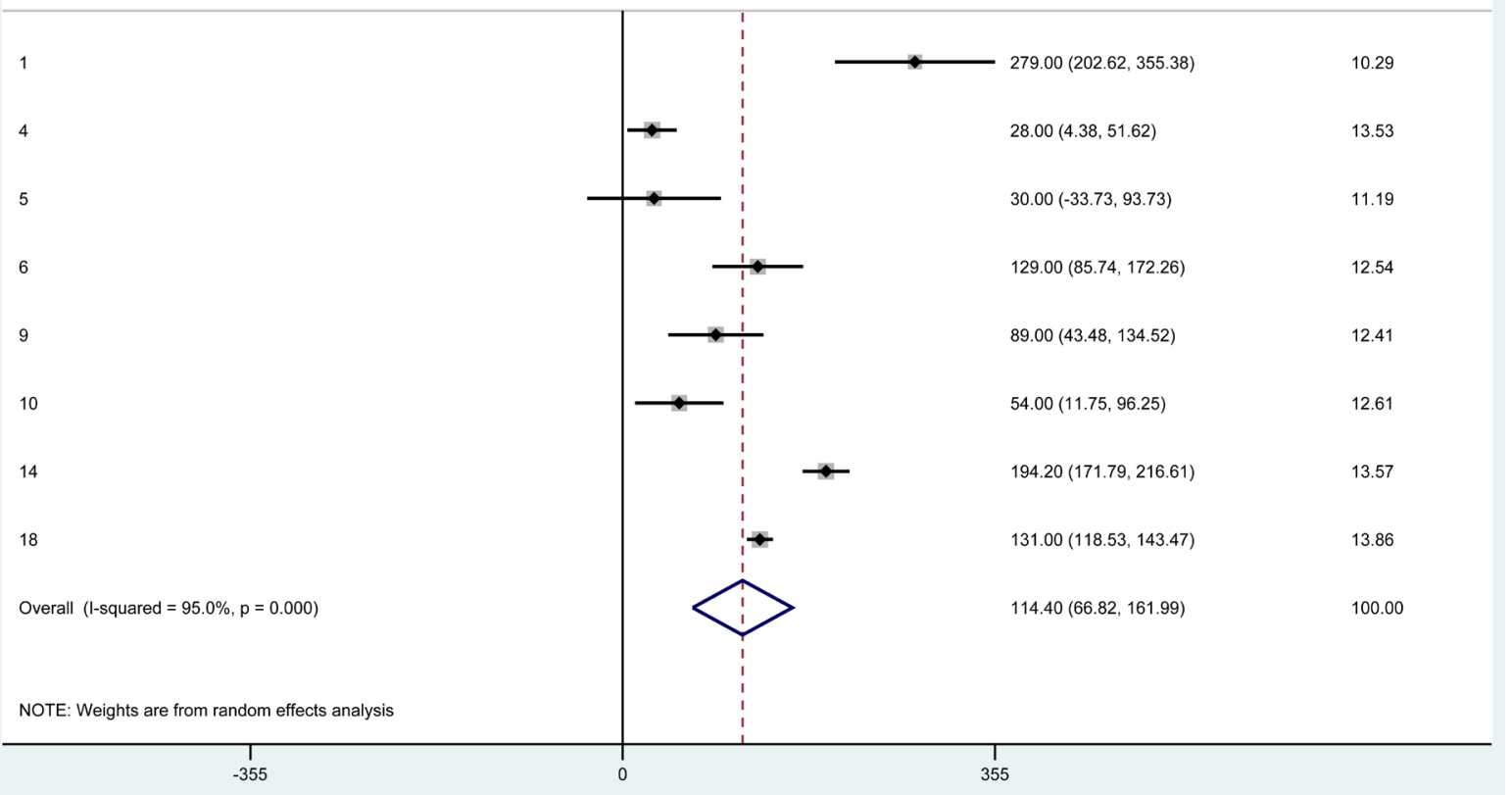

Figure 2. Forest Plot-macular thickness change post anti-VEGF for DR.
$\%$

WMD $(95 \% \mathrm{Cl})$

Weight

10.29
13.53
11.19
12.54
12.41
12.61
13.57
13.86
100.00

355 
significant $(p=0.23)$.

Table 3 reveals the compilation of most commonly reported complications from all studies. The most common complications were vitreous hemorrhage, endophthalmitis and retinal detachment. Vision threatening complications were rare but were reported regularly.

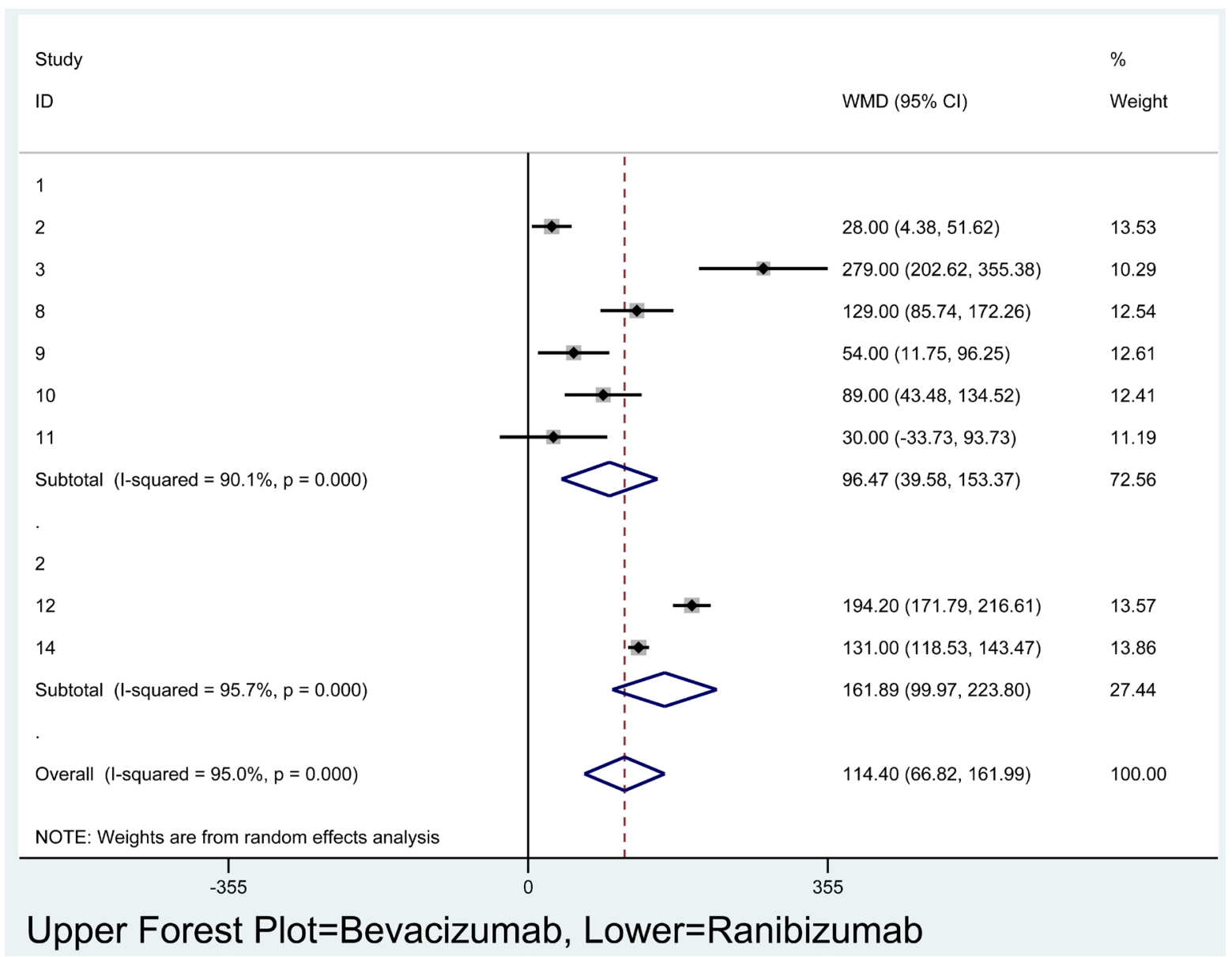

Figure 3. Macular thickness change by VEGF inhibitor.

Table 1. Baseline data.

\begin{tabular}{cccccc}
\hline & Number of Studies & Mean & Standard Deviation & Minimum Value & \multicolumn{2}{c}{ Maximum } \\
Value \\
\hline Studies & 18 & & & & \\
Patients & 17 & 187.5 & 213.5 & 31 & 691 \\
\% Female & 15 & 45.1 & 9.5 & 6.95 & 58.7 \\
HbA1c & 8 & 7.6 & 0.50 & & 8.48 \\
\hline
\end{tabular}

Table 2. Treatment characteristics.

\begin{tabular}{|c|c|c|c|c|c|}
\hline & Number of Studies & Mean & $\begin{array}{c}\text { Standard } \\
\text { Deviation }\end{array}$ & $\begin{array}{l}\text { Minimum } \\
\text { Value }\end{array}$ & $\begin{array}{l}\text { Maximum } \\
\text { Value }\end{array}$ \\
\hline Number of Eyes & 17 & 207.9 & 262.0 & 26 & 854 \\
\hline Treatment Duration (days) & 15 & 85.9 & 126.5 & 1 & 365 \\
\hline Number of Injections & 14 & 2.66 & 2.51 & 1 & 10 \\
\hline Follow-Up (days) & 17 & 317.1 & 259.6 & 7 & 730 \\
\hline
\end{tabular}


Table 3. Most commonly reported complications.

\begin{tabular}{cccccc}
\hline & $\begin{array}{c}\text { Number of Studies } \\
\text { Reporting Complication }\end{array}$ & $\begin{array}{c}\text { Mean Number of Patients } \\
\text { with Complication }\end{array}$ & $\begin{array}{c}\text { Standard } \\
\text { Deviation }\end{array}$ & Minimum & Maximum \\
\hline Vitreous Hemorrhage & 7 & 15 & 18.8 & 1 & 54 \\
Retinal Detachment & 6 & 5.2 & 9.2 & 1 & 24 \\
Endophthalmitis & 5 & 3 & & & 6 \\
\hline
\end{tabular}

Meta-regression was performed to assess the impact of age, gender, type of diabetes, study type and study quality (via Downs and Black instrument) on the outcome of macular thickness after VEGF injections. For age, gender, type of diabetes and study quality, there was no significant effect on the main outcome. For study type, the stratified sample sizes were too small to make any type of robust assessment.

\section{Conclusions}

Diabetic Retinopathy can be classified into several stages based on the level of disease severity. Non proliferative retinopathy consists of background microvascular dot hemorrhages and exudative changes termed "hard exudates". Preproliferative retinopathy consists of microvascular beading abnormalities and retinal infarcts (cotton wool spots). Proliferative retinopathy consists of fragile new vessels that can bleed into the vitreous and result in tractional retinal detachment. Broadly speaking, the main vision threatening complications are macular edema; this can occur at any stage but involves exudative thickening of the macula and neovascular bleeding which can only occur in the proliferative stage [25].

While the molecular pathogenesis of DME is not fully understood, Vascular Endothelial Growth Factor plays an important role. VEGF has a molecular weight of $46 \mathrm{kDa}$ and is a homodimer. It mediates many important physiologic processes including the development and maintenance of the vasculature, regulation of hematologic coagulation and vascular integrity through the production of nitric oxide [26] [27].

The human VEGF family comprises five related glycoproteins: the most important of which clinically is VEGFA. VEGF-A is the best characterized, and is mainly responsible for angiogenic and vasopermeability [28] [29]. All VEGF family members signal through three tyrosine kinase receptors but most of the vascular-hyperpermeability responses to VEGF-A are mediated through VEGFR-2, which is expressed by the vascular endothelium [27].

In patients with diabetic retinopathy, chronic hyperglycemia leads to the upregulation of VEGF, resulting in angiogenesis, increased vascular permeability, and the production of pro-inflammatory cytokines [29] [30]. Thickening of the basement membrane and pericyte drop out, which are key hallmarks of retinopathy will further cascade stimulation of VEGF production and result in an increased severity of diabetic retinopathy clinically [29].

Hence anti-VEGF intraocular injections have gained widespread use in retina-choroidal vascular diseases such as age related macular degeneration and diabetic retinopathy. The synthesized evidence to date from this paper reveals that both commonly used anti VEGF treatments are effective for diabetic related clinically significant macular edema. Our results do not distinguish efficacy differences amongst the two anti-VEGF treatments studied but there is a trend toward Lucentis being superior to Avastin based on macular thickness measurements. Hence, it is possible that a homogenous randomized clinical trial for diabetic macular edema could find differences amongst the two treatments for this condition.

Complications were not common and serious vision threatening complications were especially rare amongst reported papers. Less than half the papers analyzed revealed any vision threatening complications and they were rare when reported. Hence it can be concluded that the synthesis of evidence to date favors these treatments as being safe given the overall synthesized sample size was so large.

\section{Acknowledgements}

We acknowledge the funding support from AMOSO AFP Innovation Fund (Ontario, Canada) (INN12-010) for making the project possible. 


\section{References}

[1] Lutty, G.A. (2013) Effects of Diabetes on the Eye. Investigative Ophthalmology and Visual Sciences, 54, 81-87. http://dx.doi.org/10.1167/iovs.13-12979

[2] Heydari, I., Radi, V., Razmjou, S. and Amiri, A. (2010) Chronic Complications of Diabetes Mellitus in Newly Diagnosed Patients. International Journal of Diabetes Mellitus, 2, 61-63. http://dx.doi.org/10.1016/j.ijdm.2009.08.001

[3] Nicholson, B.P. and Schachat, A.P. (2010) A Review of Clinical Trials of Anti-VEGF Agents for Diabetic Retinopathy. Graefe's Archive for Clinical and Experimental Ophthalmology, 248, 915-930. http://dx.doi.org/10.1007/s00417-010-1315-z

[4] Caldwell, R.B., Bartoli, M., Behzadian, M.A., El-Remessey, A.E.B., Al-Shabrawey, M., Platt, D.H. and Caldwell, R.W. (2003) Vascular Endothelial Growth Factor and Diabetic Retinopathy: Pathophysiologic Mechanisms and Treatment Perspectives. Diabetes/Metabolism Research and Reviews, 19, 442-455. http://dx.doi.org/10.1002/dmrr.415

[5] Lang, G.E. (2012) Diabetic Macular Edema. Ophthalmologica, 227, 21. http://dx.doi.org/10.1159/000337156

[6] Michels, S., Rosenfeld, P.J., Puliafito, C.A., Marcus, E.N. and Venkatraman, A.S. (2005) Systemic Bevacizumab (Avastin) Therapy for Neovascular Age Related Macular Degeneration: Twelve-Week Results of an Uncontrolled Open-Label Clinical Study. Ophthalmology, 112, 1035-1047. http://dx.doi.org/10.1016/j.ophtha.2005.02.007

[7] Aiello, L.P., Beck, R.W., Bressler, N.M., Browning, D.J., Chalam, K.V., Davis, M., Ferris, F.L., et al. (2011) Rationale for the Diabetic Retinopathy Clinical Research Network Treatment Protocol for Center-Involved Diabetic Macular Edema. Ophthalmology, 118, 5-14. http://dx.doi.org/10.1016/j.ophtha.2011.09.058

[8] Campochiaro, P.A., Heier, J.S., Feiner, L., Gray, S., Saroj, N., Rundle, A.C., Murahasi, W.Y., et al. (2010) Ranibizumab for Macular Edema Following Branch Retinal Vein Occlusion: Six-Month Primary End Point Results of a Phase III Study. Ophthalmology, 117, 1102-1112. http://dx.doi.org/10.1016/j.ophtha.2010.02.021

[9] di Lauro, R., De Ruggiero, P., di Lauro, R., di Lauro, M.T. and Romano, M.R. (2010) Intravitrealbevacizumab for Surgical Treatment of Severe Proliferative Diabetic Retinopathy. Graefe's Archive for Clinical and Experimental Ophthalmology, 248, 785-791. http://dx.doi.org/10.1007/s00417-010-1303-3

[10] Elman, M.J., Aiello, L.P., Beck, R.W., Bressler, N.M., Bressler, S.B., Edwards, A.R., Ferris, F.L., et al.(2010) Randomized Trial Evaluating Ranibizumab plus Prompt or Deferred Laser or Triamcinolone plus Prompt Laser for Diabetic Macular Edema. Ophthalmology, 117, 1064-1077. http://dx.doi.org/10.1016/j.ophtha.2010.02.031

[11] Elman, M.J., Qin, H., Aiello, L.P., Beck, R.W., Bressler, N.M., Ferris, F.L., Glassman, A.R., et al. (2012) Intravitreal Ranibizumab for Diabetic Macular Edema with Prompt versus Deferred Laser Treatment. Ophthalmology, 119, 2312 2318. http://dx.doi.org/10.1016/j.ophtha.2012.08.022

[12] Googe, J., Brucker, A.J., Bressler, N.M., Qin, H., Aiello, L.P., Antoszyk, A., Beck, R.W., et al. (2011) Randomized Trial Evaluating Short-Term Effects of Intravitreal Ranibizumab or Triamcinolone Acetonide on Macular Edema after Focal/Grid Laser for Diabetic Macular Edema in Eyes Also Receiving Panretinal Photocoagulation. Retina, 31, 10091027. http://dx.doi.org/10.1097/IAE.0b013e318217d739

[13] Kook, D., Wolf, A., Kreutzer, T., Neubauer, A., Strauss, R., Ulbig, M., Kampik, A., et al. (2008) Long-Term Effect of Intravitreal Bevacizumab (Avastin) in Patients with Chronic Diffuse Diabetic Macular Edema. Retina, 28, 1053-1060. http://dx.doi.org/10.1097/IAE.0b013e318176de48

[14] Kook, P.E., Maier, M., Schuster, T., Feucht, N. and Lohmann, C.P. (2011) Nine-Month Results of Intravitreal Bevacizumab versus Triamcinolone for the Treatment of Diffuse Diabetic Macular Oedema: A Retrospective Analysis. Acta Ophthalmologica, 89, 769-773. http://dx.doi.org/10.1111/j.1755-3768.2009.01823.x

[15] Massin, P., Bandello, F., Garweg, J., Hansen, L., Harding, S.P., Larsen, M., Mitchell, P., et al. (2010) Safety and Efficacy of Ranibizumab in Diabetic Macular Edema (RESOLVE Study). A 12-Month, Randomized, Controlled, DoubleMasked, Multicenter Phase II Study. Diabetes Care, 33, 2399-2405. http://dx.doi.org/10.2337/dc10-0493

[16] Mehta, S., Blinder, K.J., Shah, G.K., Kymes, S.M., Schlief, S.L. and Grand, M.G. (2009) Intravitreal Bevacizumab for the Treatment of Refractory Diabetic Macular Edema. Ophthalmic Surgery Lasers and Imaging, 41, 323-329.

[17] Michaelides, M., Fraser-Bell, S., Hamilton, R., Kaines, A., Egan, C., Bunce, C., Peto, T., et al. (2010) Macular Perfusion Determined by Fundus Fluorescein Angiography at the 4-Month Time Point in a Prospective Randomized Trial of Intravitreal Bevacizumab or Laser Therapy in the Management of Diabetic Macular Edema (Bolt Study): Report 1. Retina, 30, 781-786. http://dx.doi.org/10.1097/IAE.0b013e3181d2f145

[18] Michaelides, M., Kaines, A., Hamilton, R.D., Fraser-Bell, S., Rajendram, R., Quhill, F., Boos, C.J., et al. (2010) A Prospective Randomized Trial of Intravitreal Bevacizumab or Laser Therapy in the Management of Diabetic Macular Edema (BOLT Study): 12-Month Data: Report 2. Ophthalmology, 117, 1078-1086.e2. http://dx.doi.org/10.1016/j.ophtha.2010.03.045

[19] Nguyen, Q.D., Shah, S.M., Heier, J.S., Do, D.V., Lim, J., Boyer, D., Abraham, P., et al. (2009) Primary End Point (Six 
Months) Results of the Ranibizumab for Edema of the mAcula in Diabetes (READ-2) Study. Ophthalmology, 116, 2175-2181. http://dx.doi.org/10.1016/j.ophtha.2009.04.023

[20] Nguyen, Q.D., Shah, S.M., Khwaja, A.A., Channa, R., Hatef, E., Do, D.V., Boyer, D., et al. (2010) Two-Year Outcomes of the Ranibizumab for Edema of the mAcula in Diabetes (READ-2) Study. Ophthalmology, 117, 2146-2151. http://dx.doi.org/10.1016/j.ophtha.2010.08.016

[21] Oshima, Y., Shima, C., Wakabayashi, T., Kusaka, S., Shiraga, F., Ohji, M. and Tano, Y. (2009) Microincision Vitrectomy Surgery and Intravitreal Bevacizumab as a Surgical Adjunct to Treat Diabetic Traction Retinal Detachment. Ophthalmology, 116, 927-938. http://dx.doi.org/10.1016/j.ophtha.2008.11.005

[22] Robaszkiewicz, J., Chmielewska, K., Figurska, M., Wierzbowska, J. and Stankiewicz, A. (2012) Triple Therapy: Phaco-Vitrectomy with ILM Peeling, Retinal Endophotocoagulation, and Intraoperative Use of Bevacizumab for Diffuse Diabetic Macular Edema. Medical Science Monitor, 18, 241-251. http://dx.doi.org/10.12659/MSM.882624

[23] Scott, I. and Flynn Jr., H.W. (2007) Reducing the Risk of Endophthalmitis Following Intravitreal Injections. Retina, 27, 10-12. http://dx.doi.org/10.1097/IAE.0b013e3180307271

[24] Takamura, Y., Kubo, E. and Akagi, Y. (2009) Analysis of the Effect of Intravitreal Bevacizumab Injection on Diabetic Macular Edema after Cataract Surgery. Ophthalmology, 116, 1151-1157. http://dx.doi.org/10.1016/j.ophtha.2009.01.014

[25] Ulbig, M.W., Kampik, A. and Hamilton, A.M. (1993) Diabetic Retinopathy. Epidemiology, Risk Factors and Staging. Ophthalmology, 90, 197-209.

[26] He, H., Venema, V.J., Gu, X., Venema, R.C., Marrero, M.B. and Caldwell, R.B. (1999) Vascular Endothelial Growth Factor Signals Endothelial Cell Production of Nitric Oxide and Prostacyclin through flk-1/KDR Activation of c-Src. The Journal of Biological Chemistry, 274, 25130-25135. http://dx.doi.org/10.1074/jbc.274.35.25130

[27] Foy, K.C., Miller, M.J., Moldovan, N., Carson III, W.E. and Kaumaya, P.T. (2012) Combined Vaccination with HER-2 Peptide Followed by Therapy with VEGF Peptide Mimics Exerts Effective Anti-Tumor and Anti-Angiogenic Effects in Vitro and in Vivo. Oncoimmunology, 1, 1048-1060. http://dx.doi.org/10.4161/onci.20708

[28] Papadopoulos, N., Martin, J., Ruan, Q., Rafique, A., Rosconi, M.P., Shi, E., Pyles, E.A., Yancopoulos, G.D., Stahl, N. and Wiegand, S.J. (2012) Binding and Neutralization of Vascular Endothelial Growth Factor (VEGF) and Related Ligands by VEGF Trap, Ranibizumab and Bevacizumab. Angiogenesis, 15, 171-185. http://dx.doi.org/10.1007/s10456-011-9249-6

[29] Stewart, M.W. (2012) Anti-Vascular Endothelial Growth Factor Drug Treatment of Diabetic Macular Edema: The Evolution Continues. Current Diabetes Reviews, 8, 237-246. http://dx.doi.org/10.2174/157339912800840488

[30] Bhagat, N., Grigorian, R.A., Tutela, A. and Zarbin, M.A. (2009) Dibetic Macular Edema: Pathogenesis and Treatment. Survey of Ophthalmology, 54, 1-32. http://dx.doi.org/10.1016/j.survophthal.2008.10.001 